\title{
MOLECULAR CHARACTERISATION OF MYCOPLASMA GALLISEPTICUM ISOLATES FROM IRAN IN THE PERIOD 2012-2017
}

\author{
Hassan NOROUZIAN $^{1 *}$, Ghasem FARJANIKISH ${ }^{2}$ and Hossein HOSSEINI ${ }^{3}$ \\ ${ }^{1}$ Department of Clinical Sciences and ${ }^{2}$ Department of Pathobiology, Faculty of \\ Veterinary Medicine, Lorestan University, P. O. Box 465, Khorramabad, Iran; \\ ${ }^{3}$ Department of Clinical Sciences, Faculty of Veterinary Medicine, \\ Islamic Azad University, Karaj, Iran
}

(Received 9 January 2019; accepted 31 July 2019)

\begin{abstract}
Mycoplasma gallisepticum (MG) causes chronic non-fatal diseases in the poultry industry with a remarkable decrease in feed consumption, egg production and other production indices. To study the genetic characteristics of MG isolates in commercial and backyard poultry flocks, 21 positive samples from different regions of Iran were analysed in the period 2012-2017. Typical macroscopic and histopathological lesions of the upper respiratory tract and lungs were found, similar to those reported by other researchers. A 298-361 bp part of the $m g c 2$ gene was sequenced and analysed. Based on the phylogenetic analysis, the Iranian MG isolates fell into four distinct subgroups. The nucleotide difference between subgroups 1 and 4 was remarkable (91.6-94.9\%). A 22-amino-acid insertion was present in two of the studied MG isolates, not observed in other vaccine and standard MG strains. The Iranian Veterinary Organisation (IVO) should consider the observed diversity of prevalent MG isolates from both commercial and backyard flocks in designing the strategy for controlling MG. More studies are needed to understand modifications in MG antigenicity and pathogenicity because of the observed genetic variations.
\end{abstract}

Key words: Mycoplasma gallisepticum, poultry, phylogenetic analysis, $m g c 2$, histopathology

Mycoplasma gallisepticum (MG) infections commonly cause a chronic, non-fatal respiratory disease in chickens and turkeys. However, decrease in weight gain and egg production as well as increased mortality and poor hatchability make MG infections very costly for the poultry industry. The disease is commonly transmitted among closely related bird species (Raviv and Ley, 2013). Moreover, because of vertical transmission, $\mathrm{MG}$ is an important problem in the poultry production chains. ${ }^{*}$ Corresponding author; E-mail: noroozianh@yahoo.com; Phone:0098-916-159-6032;
Fax: 0098-66-331-20109 
MGC2 is one of the cytadhesin proteins of MG that mediates attachment to cells (Raviv and Ley, 2013). Because of recognition by the host's immune system, it is under a high and constant selection pressure (Hong et al., 2005). The genetic variability of the $m g c 2$ gene has previously been documented (Hnatow et al., 1998; Ferguson et al., 2005; Gharaibeh et al., 2011; Eissa et al., 2014). For epizootiological studies, it is necessary to apply reliable methods for the differentiation of MG strains, so that one will be able to trace the source of the pathogen in outbreaks (Ferguson et al., 2005). Gene-targeted sequencing (GTS) analysis of portions of MG cytadhesin genes, like $p v p A, g a p A$ and $m g c 2$, has been confirmed to be a better method than RAPD, because of the reproducibility of results and a better discriminatory power and, therefore, it serves as a common method for studies on MG (Moretti et al., 2013; Tyler et al., 1997). Except for the $p v p A$ gene, the discriminatory power of $m g c 2$ GTS analysis is higher than that of other cytadhesin genes (Ferguson et al., 2005).

In recent years, poultry meat and egg production has increased tremendously in Iran (Rahimi, 2013). In addition, like in other developing countries, there is a large backyard poultry population in Iran, supplying the domestic market with poultry meat and eggs. Nevertheless, the lack of efficient vaccination programs and biosecurity policies make backyard poultry an important reservoir for MG (Kelly et al., 1994). A study on the 1999 outbreak of MG in North Carolina commercial poultry showed that backyard flocks were important reservoirs for the disease (Ley et al., 2003). In spite of a national control program, MG infection is present at a relatively high rate in commercial poultry farms of Iran (Haghighi-Khoshkhoo et al., 2011; Seifi and Shirzad, 2012).

To date, few studies have been conducted on the genetic characterisation of MG strains in commercial and backyard poultry flocks of Iran (Loolmani et al., 2014; Rasoulinezhad et al., 2017). The aim of this study was to evaluate MGaffected commercial and backyard poultry flocks from pathological and molecular points of view.

\section{Materials and methods}

\section{Sampling and necropsy of birds}

In this study, a total of 1348 swab samples from 130 flocks of commercial and backyard chickens and turkeys affected by respiratory diseases in the period 2012-2017 were analysed. Swabs were taken from the choanal cleft, left to dry and then stored at $-20^{\circ} \mathrm{C}$. Usually, 10-20 swabs were collected from one flock, except for small backyard flocks in which less than 10 swabs could be obtained. Every 3-5 swabs were pooled in one microtube and tested for the detection of MG using PCR. Flocks with at least one positive result were considered MG positive. No MG vaccine had been administered to the backyard poultry and broiler chickens studied. 
At necropsy, the appropriate tissues were collected, fixed in $10 \%$ neutral buffered formalin, processed using a routine histological technique and embedded in paraffin. Sections of $5 \mu \mathrm{m}$ thickness were made from the paraffin blocks and were stained with haematoxylin and eosin. All procedures were conducted in agreement with the National Institutes of Health Guide for the Care and Use of Laboratory Animals.

\section{Detection of $M G$ infection by PCR test}

DNA was extracted from pooled swab samples using CinnaPure-DNA (CinaClon, Iran) according to the manufacturer's instructions. Extracted DNA was stored at $4{ }^{\circ} \mathrm{C}$ for immediate use or at $-20^{\circ} \mathrm{C}$ for later use. All samples were subjected to PCR, using specific primers for MG (García et al., 2005). A pair of oligonucleotide primers, mgc2-2F (5'-CGC AAT TTG GTC CTA ATC CCC AAC A-3') and mgc2-2R (5'-TAA ACC CAC CTC CAG CTT TAT TTC C-3') was used for the detection of MG. The amplification was carried out in a total reaction volume of $20 \mu \mathrm{l}$, consisting of $2 \mu \mathrm{l}$ of $10 \times$ PCR buffer, $1 \mu \mathrm{l}$ of mixed primer $(25 \mu \mathrm{M}), 0.2 \mu \mathrm{l}$ of $10 \mathrm{mM}$ dNTP, $1 \mu \mathrm{l}$ of $50 \mathrm{mM} \mathrm{MgCl} 2,0.25 \mu \mathrm{l}$ of Taq DNA polymerase $(5 \mathrm{U} / \mu \mathrm{l})$, and $2 \mu \mathrm{l}$ of template DNA. The amplification reaction was performed in a T100 Thermal Cycler (Bio-Rad, United States) as follows: $94{ }^{\circ} \mathrm{C}$ for $3 \mathrm{~min}$, followed by 40 cycles of $94{ }^{\circ} \mathrm{C}$ for $10 \mathrm{sec}, 56^{\circ} \mathrm{C}$ for $10 \mathrm{sec}, 72{ }^{\circ} \mathrm{C}$ for $10 \mathrm{sec}$, and a final extension at $72{ }^{\circ} \mathrm{C}$ for $5 \mathrm{~min}$. The amplification products were electrophoresed on agarose gel (2\%) in TBE buffer. Gels were run for $1 \mathrm{~h}$ at $90 \mathrm{~V}$, stained with ethidium bromide and visualised under UV light. DNA fragments of the expected length were extracted and purified with the GeneJET Gel Extraction Kit (Thermo Scientific, Canada). A total number of 21 purified samples were sent for nucleotide sequencing (Bioneer, South Korea). The acquired accession numbers of MG sequences from GenBank are presented in Table 1.

\section{Genetic analysis}

Pairwise nucleotide and deduced amino acid sequence alignments were performed with the Clustal W (Mega program, version 5) on the obtained sequences and some other sequences from reference and vaccine MG strains available in GenBank. Sequence similarity and phylogenetic relationships of different MG isolates were also studied with the Mega program, version 5.

\section{Results}

\section{Sampling and necropsy of birds}

A total of 21 flocks (16\%) were positive for MG. Sick birds showed clinical signs of respiratory infection including nasal and ocular discharge, coughing, 
sneezing and swelling of the infraorbital sinus. In layer flocks, delay and decrease in egg production and a slight increase in mortality rate were regarded as the prominent symptoms.

Table 1

Characteristics of the Mycoplasma gallisepticum isolates studied

\begin{tabular}{lcccc}
\hline Isolate & Location & Host & Sample type & Accession number \\
\hline L744/13 & Kerman & Layer & Tracheal swab & KY491037 \\
L1245/15 & Mazandaran & Broiler breeder & Tracheal swab & KY421188 \\
L1267/15 & Khorasan & Layer & Tracheal swab & KY491039 \\
L1789/16 & Khorasan & Backyard chicken & Tracheal swab & KY012362 \\
L1794/16 & Golestan & Broiler breeder & Tracheal swab & KY421187 \\
L419.3/12 & Kermanshah & Broiler & Tracheal swab & KY491031 \\
L1043.1/14 & Yazd & Broiler & Tracheal swab & KY491036 \\
L1134.7/16 & Gilan & Broiler breeder & Tracheal swab & KY491032 \\
L1245.4/15 & Lorestan & Layer & Tracheal swab & KY491038 \\
L1277.8/15 & Mazandaran & Broiler breeder & Tracheal swab & KY491033 \\
L1298.2/15 & Lorestan & Backyard turkey & Tracheal swab & KX650154 \\
L1651.28/16 & Markazi & Layer & Tracheal swab & KY491034 \\
L1298.3/15 & Lorestan & Backyard turkey & Tracheal swab & KX650155 \\
L1646.2/16 & Lorestan & Backyard turkey & Tracheal swab & KX650156 \\
L1860.2/16 & Qazvin & Backyard chicken & Tracheal swab & KY491035 \\
L1940.1/17 & Tehran & Broiler breeder & Tracheal swab & KY491030 \\
L1940.3/17 & Qazvin & Broiler & Tracheal swab & KY491029 \\
L2013.49/17 & Khuzestan & Broiler & Tracheal swab & MG428414 \\
L2013.52/17 & Kermanshah & Broiler & Tracheal swab & MG428415 \\
L2013.48/17 & Tehran & Broiler & Tracheal swab & MG428413 \\
L2013.4/17 & Khorasan & Broiler & Tracheal swab & MG428412 \\
\hline & & & & \\
\hline
\end{tabular}

Macroscopic lesions were characterised as frothy exudates in the nasal and paranasal passages, trachea, bronchi, and air sacs. Sinusitis was observed both in turkeys and chickens. In turkeys, sinusitis was characterised by severe sinus swelling with viscous honey-coloured exudates in the infraorbital sinuses (Fig. 1A). The trachea and lungs showed evidence of congestion, haemorrhages and fibrinous exudates. Accumulation of serofibrinous to caseous exudates was noticed in the walls of air sacs. The liver and heart were pale, and perihepatitis and pericarditis with fibrinopurulent exudate covering the organs were observed.

\section{Histopathology}

The most consistent histopathological alterations were observed in the upper respiratory tract and lungs. Histopathological examination revealed marked thickening of the mucous membranes of sinuses associated with epithelial hyperplasia, as well as mononuclear cell infiltration and hyperplasia of the mucous glands. The sinus was abundantly filled with diffuse mucopurulent exudates (Fig. 1B). In the trachea and primary bronchi, epithelial cells in many parts lost their 
cilia and the tracheal epithelium was hyperaemic, degenerated and necrotic, and the number of mucosal glands decreased. Epithelial cells and cilia in some areas were covered with a thick layer of fibrinopurulent exudate with intensive and diffuse infiltrations by heterophils and lymphocytes (Fig. 1C). The lungs showed congestion, haemorrhages, accumulation of fibrin exudates, cellular debris, pneumonic areas and leukocytic infiltrations (Fig. 1D). The liver and heart tissues showed diffuse mononuclear inflammatory cell infiltration and distension of the vessels.
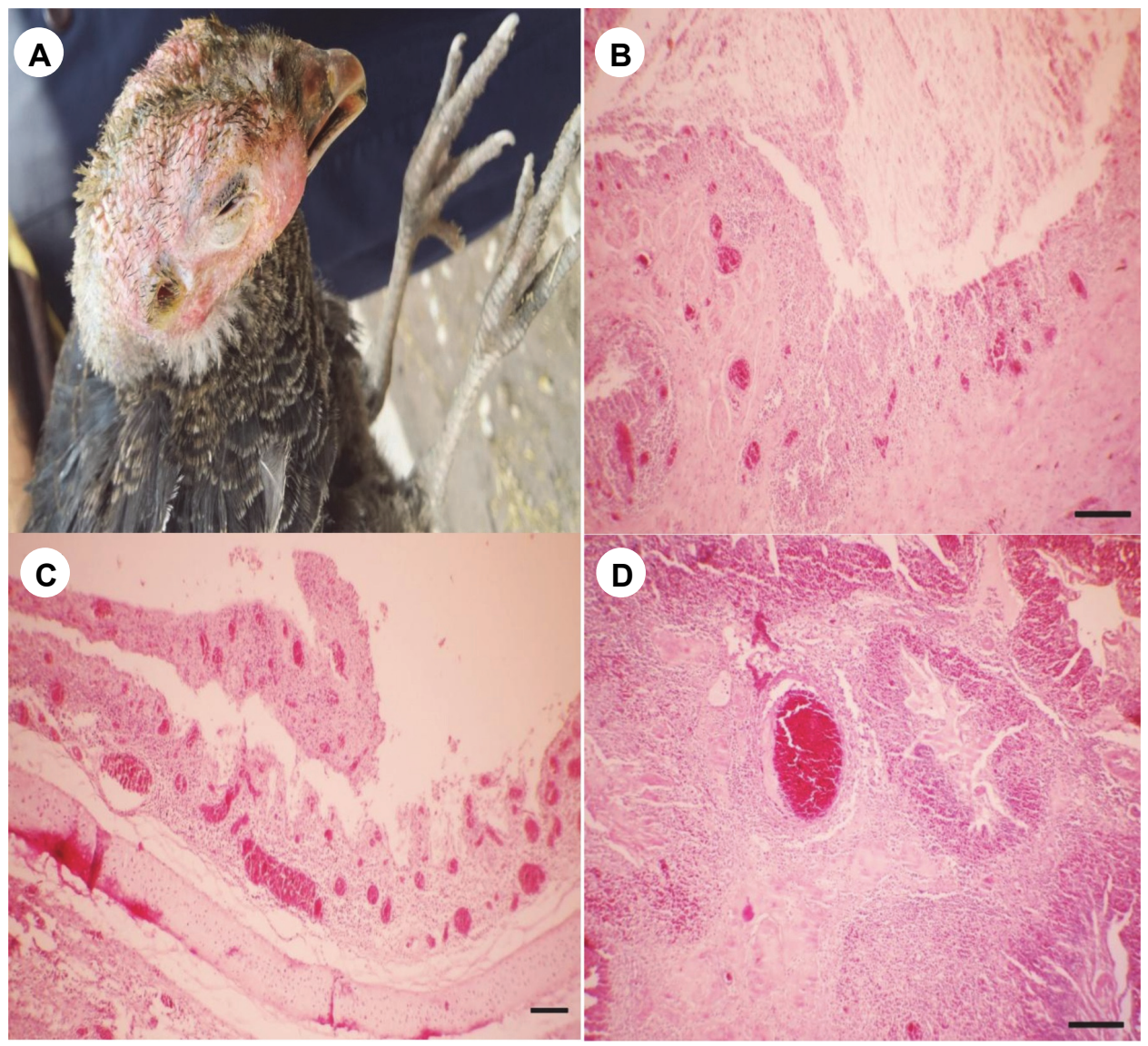

Fig. 1A. Turkey. Sinusitis characterised by severe sinus swelling in the infraorbital sinus. $1 B$. Infraorbital sinus. Thickening of the epithelial membrane associated with epithelial hyperplasia, hyperaemia and mononuclear cell infiltration. The sinus is abundantly filled with a diffuse mucopurulent exudate. Haematoxylin and eosin (HE), bar $=200 \mu \mathrm{m}$. 1C. Trachea. The epithelium is hyperaemic, degenerated and infiltrated with inflammatory cells. Thick layer of fibrinopurulent exudate with intensive and diffuse infiltration with heterophils and lymphocytes cover the epithelium (HE, bar = $100 \mu \mathrm{m}) .1 D$. Lungs. Interstitial pneumonia and accumulation of diffuse fibrinopurulent exudates and congestion are observed $(\mathrm{HE}, \mathrm{bar}=100 \mu \mathrm{m})$ 


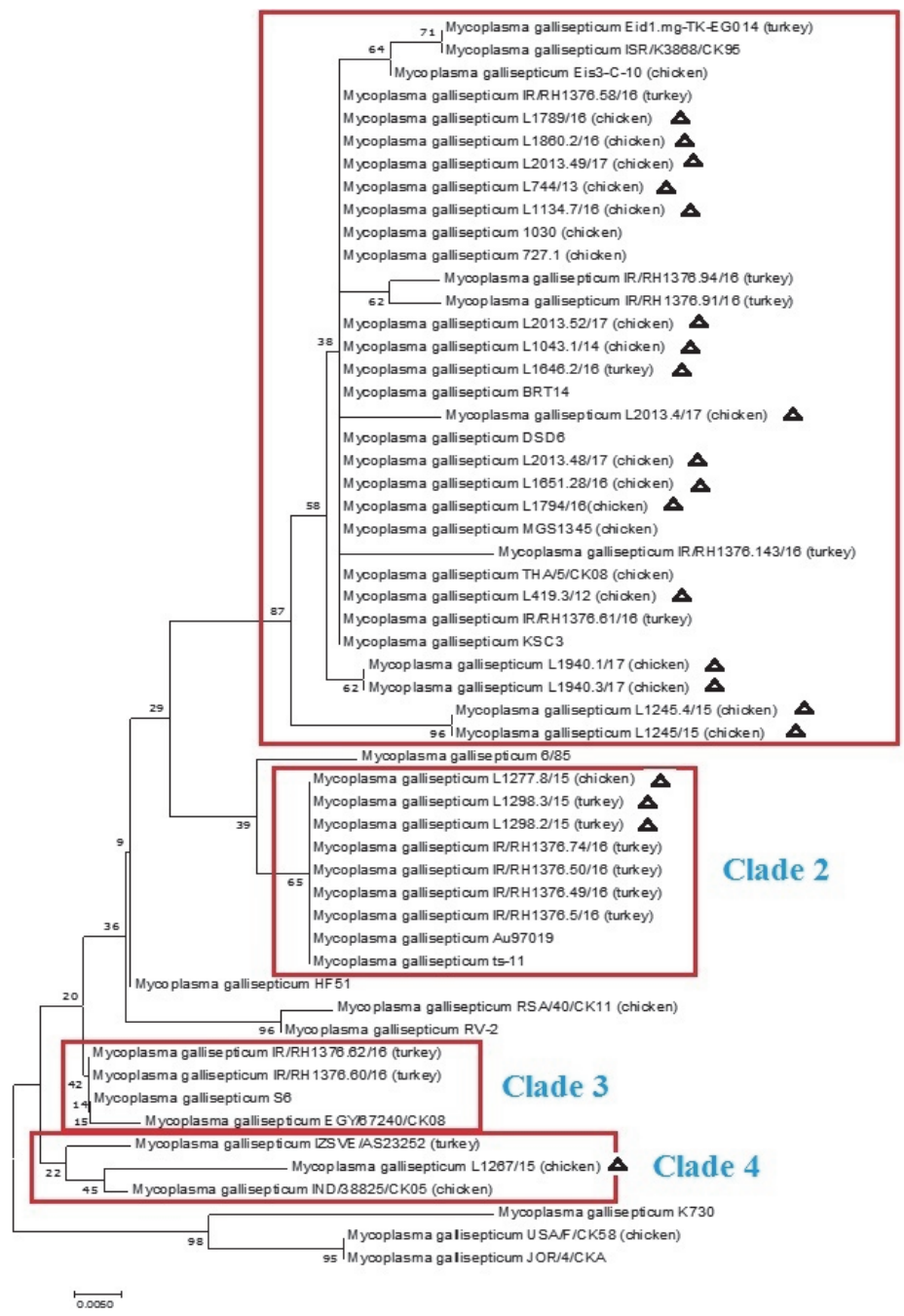

Clade 1

Fig. 2. Phylogenetic tree of the studied Mycoplasma gallisepticum (MG) isolates and other Iranian, reference and vaccine strains. The tree constructed by the Clustal W method is based on the nucleotide sequence of mgc2. Numbers at the nodes indicate the bootstrap confidence values calculated using 1000 bootstrapping replicates. Sequenced isolates are indicated with triangles. All viruses sequenced in this study and the other sequences can be found in GenBank; MG Eid1.mg-TK-EG014 (KP691072), MG ISR/K3868/CK95 (HQ143377), MG strain Eis3-C-10 (HQ591355), MG strain IR/RH1376.58/16 (KY651220), MG clone 1030 (GQ436786), MG clone 727.1 (GQ436784), MG strain IR/RH1376.94/16 (KY651226), MG strain IR/RH1376.91/16 (KY651225), MG strain BRT14 (AY556291), MG strain DSD6 (AY556292), MG MGS1345 (KP300762), MG strain IR/RH1376.143/16 (KY651227), MG THA/5/CK08 (KC247894), MG IR/RH1376.61/16 (KY651222), MG KSC3 (AY556294), MG 6/85 (KP318741), MG IR/RH1376.74/16 (KY651224), MG IR/RH1376.50/16 (KY651219), MG strain IR/RH1376.49/16 (KY651218), MG strain IR/RH1376.5/16 (KY651217),

MG Au97019 (AY556302), MG ts11 (AY556232), MG HF51 (AY556233), MG RSA/40/CK11 (KC247872), MG RV-2 (EU939449), MG IR/RH1376.62/16 (KY651223), MG IR/RH1376.60/16 (KY651221), MG S6 (JQ770177), MG EGY/67240/CK08 (HQ143372), MG strain IZSVE/AS23252 (KM107807), MG strain IND/38825/CK05 (KC247892), MG K730 (AY556236), MG USA/F/CK58 (KC247897), MG JOR/4/CKA (HQ143378) 


\section{Genetic analysis}

The size of the PCR amplicons in most isolates was $288 \mathrm{bp}$. Because of an insertion, this was increased to $361 \mathrm{bp}$ in two isolates (L744/13 and L1043.1/14). Nucleotide sequences of the partial $m g c 2$ of Iranian isolates were classified in four clades (Fig. 2). Within each clade, the degree of similarity was $97.6-100 \%$. The four clades were different from each other, with $91.6-98.3 \%$ degree of similarity. Particularly the difference between subgroups 1 and 4 was remarkable (91.6-94.7\%) (Table 2). The degree of nucleotide similarity between the studied MG isolates and strains from other countries was $90.1 \%$ for $\mathrm{K} 730$ and $100 \%$ for BRT14, THA5CK08, MGS1345, etc. Moreover, the degree of similarity between the studied MG isolates and vaccine strains was $94.4-98.4 \%$ (for vaccine strain $6 / 85$ ) and $95.5-100 \%$ (for vaccine strain ts- 11 ).

\section{Table 2}

Homology comparison of the $m g c 2$ nucleotide and amino acid sequences of different subgroups in the studied Iranian isolates

\begin{tabular}{lcccc}
\hline & Subgroup 1 & Subgroup 2 & Subgroup 3 & Subgroup 4 \\
\hline Subgroup 1 & $97.6-100 \%$ & $94.7-97.9 \%$ & $95.4-98.3 \%$ & $91.6-94.7 \%$ \\
Subgroup 2 & - & $100 \%$ & $97.9 \%$ & $96.1 \%$ \\
Subgroup 3 & - & - & $100 \%$ & $96.1 \%$ \\
Subgroup 4 & - & - & - & $100 \%$ \\
\hline
\end{tabular}

When comparing the deduced amino acid sequences of the studied MG isolates, there was a complete similarity between some isolates from Israel, India and Thailand with most of the studied MG isolates. The amino acid similarity among the sequenced isolates was $90.8-100 \%$. There were four amino acid residues (11I, 22P, 62I and 90L) among most of the studied MG isolates which are unique to the Middle East isolates. A 22-amino-acid insertion was present in two studied MG isolates (L1043.1/14 and L744/13), which was also observed in another Iranian isolate (727.1) and in IZSVE/AS23252, but not in vaccine and standard MG strains (Fig. 3).

\section{Phylogenetic analysis}

Using BLAST [http://blast.ncbi.nlm.nih.gov/Blast.cgi], the nucleotide sequences of MG isolates, obtained in this study, were evaluated to find other MG isolates with the highest similarity. Based on the phylogenetic analysis, all Iranian MG isolates fell into four distinct clades. Clade 1 consisted of seventeen sequenced MG isolates, and mostly of isolates from the Middle East, including Egypt and Israel, as well as isolates from India and Thailand. Clade 2, in which three sequenced isolates were placed, was next to the Australian isolates and the ts-11 vaccine strain (99-99.3\% similarity) and more distant from isolates from 
the neighbouring countries (91.5-97.3\% similarity). Clade 3 contained two Iranian isolates, an Egyptian isolate and the S6 standard isolate. In Clade 4, there was only one sequenced isolate which was distant from the other Iranian isolates and stood alone in a separate clade (Fig. 2).

\section{Discussion}

Only few studies are available on the characterisation of MG field strains circulating in Iran (Loolmani et al., 2014; Rasoulinezhad et al., 2017). In our study, the genetic characteristics of $21 \mathrm{MG}$ isolates obtained from commercial and backyard chickens and turkeys from different geographic locations in Iran in the period 2012-2017 were evaluated using sequence analysis of the partial $m g c 2$ gene. Considering the distinct geographical regions and time periods, the MG isolates demonstrated a wide variation (91.6-100\%) of nucleotide sequence homology. However, there was a complete similarity among isolates obtained from different farms in different geographical regions, revealing a high prevalence of similar MG strains in poultry farms of Iran. Such a complete similarity among MG isolates obtained from different farms has previously been reported (Abdelwhab et al., 2011; Gharaibeh et al., 2011; Eissa et al., 2014). This could be justified based on the vertical transmission of MG from a breeder flock to many broiler or layer flocks in different geographical locations.

A high nucleotide similarity between isolates from backyard turkey and commercial poultry flocks (e.g. isolates L1298.3/15 and L1277.8/15 in Clade 2) confirmed the probability of a two-way transmission between those two sectors of the poultry industry. Recently, the Ministry of Agriculture in Iran has provided backyard poultry for customers, mainly to increase the poultry production potential of the rural sector and to generate self-employment in rural areas. Cultivation of backyard poultry, which is mostly under poor surveillance by the Iranian Veterinary Organisation (IVO), can be a permanent threat to the eradication plan of $M G$ in the commercial poultry industry. Identification of MG genotypes in backyard poultry is needed to improve control strategies of the disease in the commercial poultry sector.

In the phylogenetic analysis, the studied isolates were divided into four separate clades with a relatively high diversity among them. Two most important factors typically cause isolates to be placed in different subgroups: geographical distance and isolation time. Nevertheless, the immigration of wild birds and the poultry trade may have a significant role in MG transmission in distant geographical regions (Ferguson et al., 2005), and therefore may affect the classification of isolates in the phylogenetic tree. Gharabieh et al. (2011) showed that MG isolates from Jordan during two time periods from 2004 to 2008 were classified as two separate groups, mainly based on the isolation time. In our study, the effect of time on the classification of isolates was not evident. In the study of Eissa 


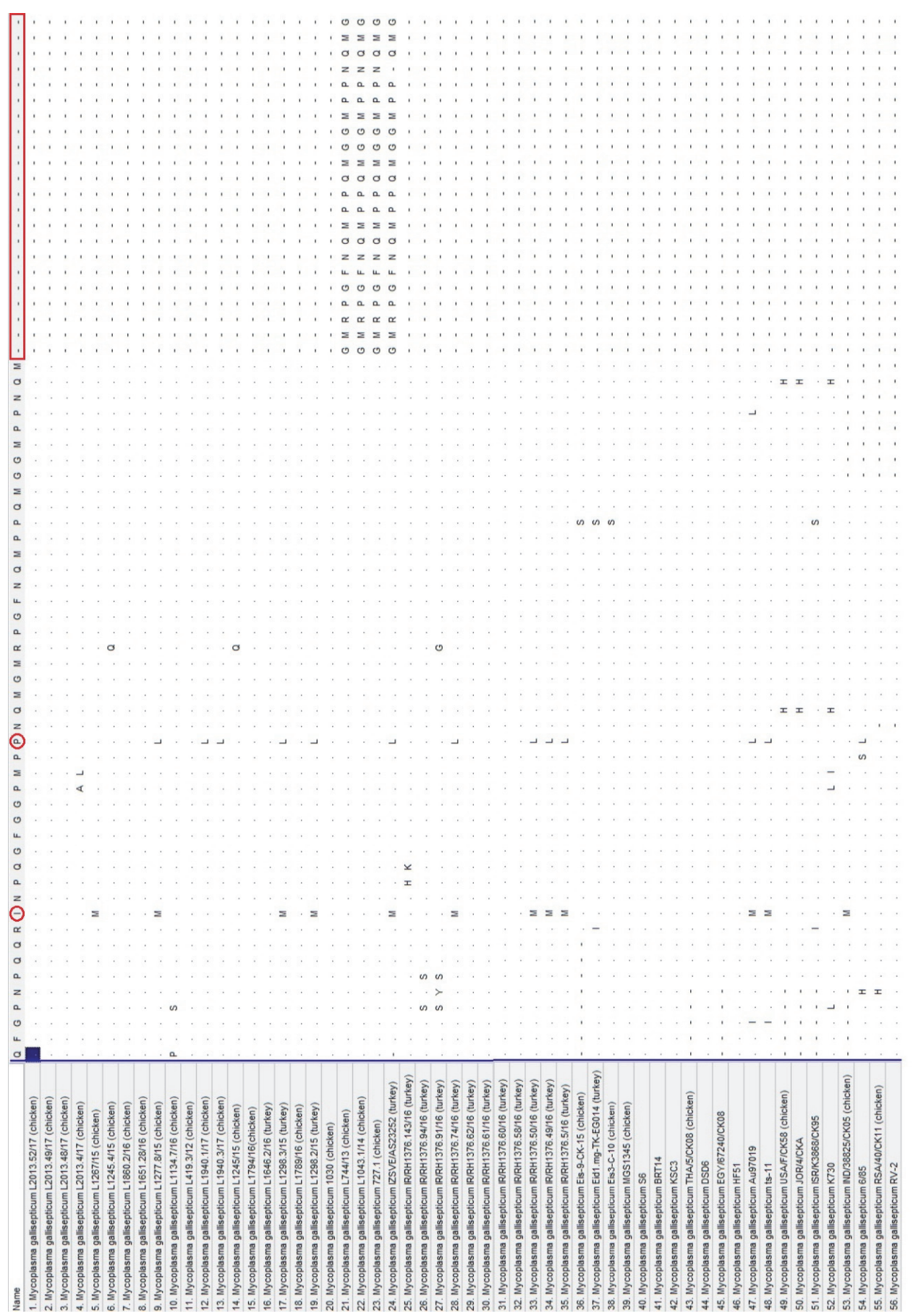




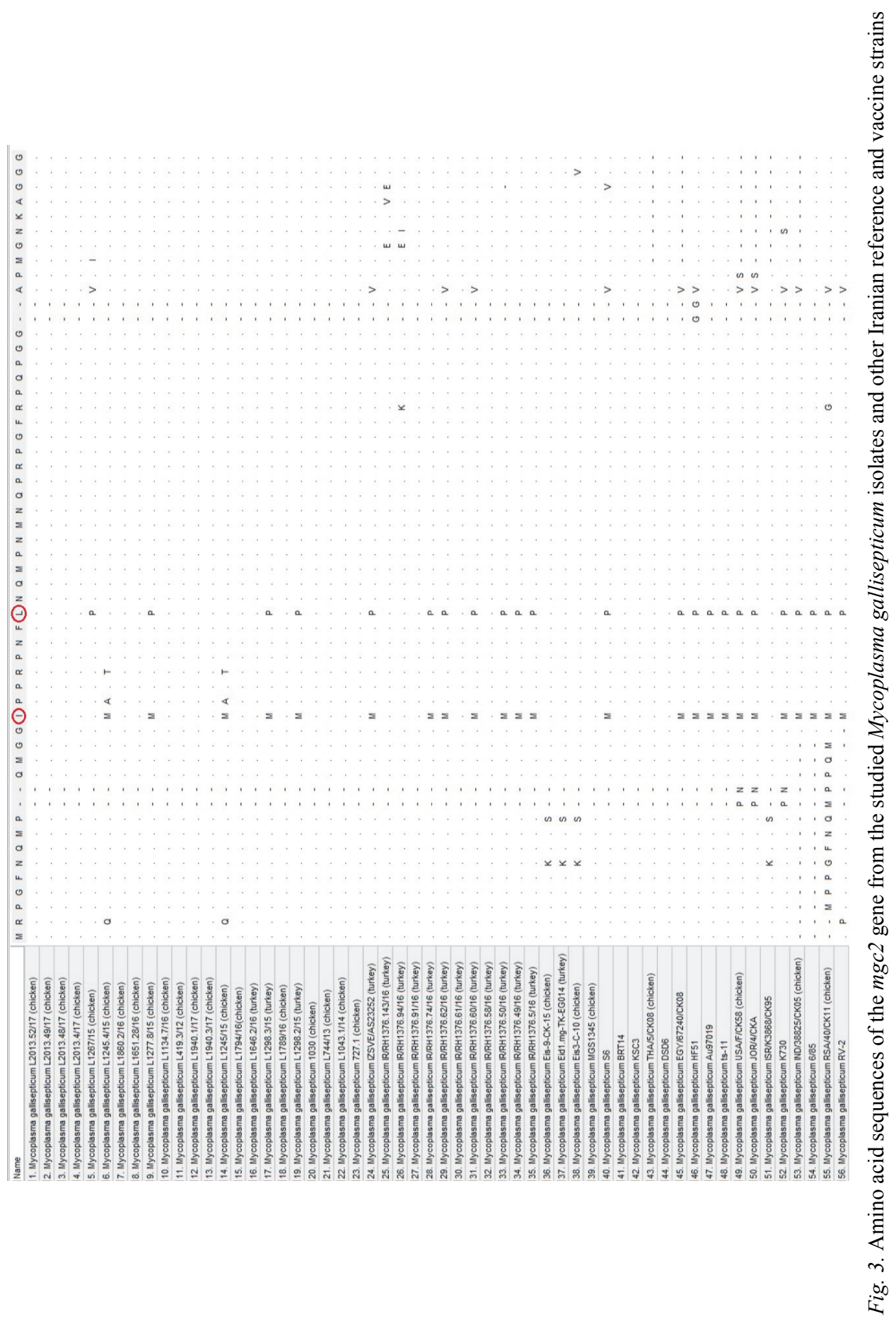


et al. (2014), although most Egyptian MG isolates were closely related to each other in a separate clade far from the US and Australian isolates, some isolates were related to the $\mathrm{F}$ vaccine strain, which has been used in Egypt for the vaccination of commercial poultry. The results reported by Younis et al. (2018) also confirmed the close relationship among Egyptian MG strains isolated from broilers with respiratory diseases. On the other hand, the results by Ferguson et al. (2005) showed that in the phylogenetic analysis of $m g c 2$ and other cytadhesin genes, in spite of some minor sub-clustering, all MG isolates from one country were located close to one another. They showed that the US isolates from known poultry outbreaks were closely related to vaccine strains, which is probably due to the wide administration of live MG vaccines (Ferguson et al., 2005). No MG vaccine was being used in Iranian poultry farms. In our study, the isolates of Clade 2 were close to the Australian isolates and the ts- 11 vaccine strain. The phylogenetic relationship of the Clade 2 isolates to the ts- 11 vaccine strain is unique among the Middle Eastern isolates. Anyway, more studies in different time periods and different geographical regions of Iran are needed to draw comprehensive epizootiological conclusions.

Two sequences with a 63-nucleotide insertion across the $m g c 2$ gene were observed in this study. The insertion is present in another Iranian isolate (727.1) and also in isolate IZSVE/AS23252 but not in any vaccine and standard MG strains. Because of insertion and/or deletion, gene size polymorphism is common in MG strains (Hnatow et al., 1998). The vaccine strains (6/85 and F strains) and isolates from India, Israel and South Africa have deletions across the $m g c 2$ carboxy-terminus (Hnatow et al., 1998; Moretti et al., 2013). Short nucleotide variations in the $m g c 2$ gene are difficult to detect on usual stained agarose gels and are not considered as a suitable method for differentiating among MG isolates (Ferguson et al., 2005), although such insertions and/or deletions may lead to significant changes in the antigenic domains of $m g c 2$. Four major amino acid residues (11I, 22P, 62I and 69L) in most of the studied MG isolates were a fingerprint for the Middle Eastern MG isolates. Because $m g c 2$ is projected on the surface of $\mathrm{MG}$, the amino acid specific changes in the $m g c 2$ gene may alter not only surface exposure and hydrophilicity but also the tertiary structure of the protein (Hnatow et al., 1998). This is a trick by which MG is able to change the expression of surface antigens and thereby to alter the 'antigenic profile' presented to the host's immune system (Bencina, 2002). Another notifiable amino acid residue in one of the isolates (L1267/15) was $113 \mathrm{~V}$, which is a fingerprint of the $\mathrm{F}$ vaccine strain. Eissa et al. (2014) also reported that residue in Egyptian MG isolates.

The administration of antibiotics like tylosin is common as a control method of MG infection (Abdelwhab et al., 2011). However, the application of antibiotics will not eliminate the organism and, on the other hand, it enhances the selective pressure of the host immune system on MG to make amino acid changes in surface antigens like $m g c 2$. The remarkable sequence variation in the $m g c 2$ 
gene of Iranian MG isolates might be due to the widespread use of antibiotics in Iranian breeder farms. When the complete eradication of MG is difficult to attain, vaccination with live vaccines is utilised as an alternative control strategy (Whithear, 1996; Kleven, 1997). The IVO should consider the diversity of prevalent MG strains in the use of vaccines for controlling MG. In South Africa, the presence of MG genotypes different from common vaccines was considered as an explanation for the failure of MG vaccination in the poultry industry (Moretti et al., 2013).

Our study suggested a high genetic variability in MG isolates from commercial and backyard poultry farms of Iran, which is important in designing the eradication strategy of the disease and also the use of vaccination to achieve that goal. Extensive backyard poultry rearing without even the lowest level of biosecurity should be considered an important item in the eradication plan.

\section{Acknowledgement}

This research was financially supported by the Deputy of Research, Lorestan University, Khorram Abad, Iran.

\section{References}

Abdelwhab, E. M., Abdelmagid, M. A., El-Sheibeny, L. M., El-Nagar, H. A., Arafa, A., Selim, A., Nasef, S. A., Aly, M. M. and Hafez, H. M. (2011): Detection and molecular characterization of Mycoplasma gallisepticum field infection in TS-11-vaccinated broiler breeders. J. Appl. Poult. Res. 20, 390-396.

Bencina, D. (2002): Haemagglutinins of pathogenic avian mycoplasmas. Avian Pathol. 31, 535-547.

Eissa, S. I., Metwally, A. M., Hashem, Y. M., Khalifa, R. A. and Refaie, M. K. (2014): Molecular comparative analysis of Mycoplasma gallisepticum field and vaccine strains in Egypt. Eur. J. Vet. Med. 2014, 9.

Ferguson, N. M., Hepp, D., Sun, S., Ikuta, N., Levisohn, S., Kleven, S. H. and Garcia, M. (2005): Use of molecular diversity of Mycoplasma gallisepticum by gene-targeted sequencing (GTS) and random amplified polymorphic DNA (RAPD) analysis for epidemiological studies. Microbiol. 151, 1883-1893.

Garcia, M., Ikuta, N., Levison, S. and Kleven, S. H. (2005): Evaluation and comparison of various PCR methods for detection of Mycoplasma gallisepticum infection in chickens. Avian Dis. 49, 125-132.

Gharaibeh, S., Laibinis, V., Wooten, R., Stabler, L. and Ferguson-Noel, N. (2011): Molecular characterization of Mycoplasma gallisepticum isolates from Jordan. Avian Dis. 55, 212-216.

Haghighi-Khoshkhoo, P., Akbariazad, G., Roohi, M., Inanlo, J., Masoumi, M. and Sami-Yousefi, P. (2011): Seroprevalence of Mycoplasma gallisepticum and Mycoplasma synoviae infection in the commercial layer flocks of the centernorth of Iran. Afr. J. Microbiol. Res. 5, $2834-2837$.

Hnatow, L. L., Keeler, C. L., Tessmer, L. L., Czymmek, K. and Dohms, J. E. (1998): Characterization of MGC2, a Mycoplasma gallisepticum cytadhesin with homology to the Mycoplasma pneumoniae 30-kilodalton protein P30 and Mycoplasma genitalium P32. Infect. Immun. 66, 3436-3442. 
Hong, Y., García, M., Levisohn, S., Savelkoul, P., Leiting, V., Lysnyansky, I., Ley, D. H. and Kleven, S. H. (2005): Differentiation of Mycoplasma gallisepticum strains using amplified fragment length polymorphism and other DNA-based typing methods. Avian Dis. 49, 43-49.

Kelly, P. J., Chitauro, D., Rohde, C., Rukwava, J., Majok, A., Davelaar, F. and Mason, P. R. (1994): Diseases and management of backyard chicken flocks in Chitungwiza, Zimbabwe. Avian Dis. 38, 626-629.

Kleven, H. (1997): Changing expectations in the control of Mycoplasma gallisepticum. Acta Vet. Hung. 45, 299-305.

Ley, D., Martinez, A. and Vaillancourt, J. (2003): Mycoplasma gallisepticum outbreaks in North Carolina, 1999-2001: lessons learned from the tail end of the epidemic curve [Internet]. [cited Sep 2012]. Available from: http://www.cvm.ncsu.edu/dphp/phm/documents/ LeyAVMA2003.pdf

Loolmani, F. S., Pourbakhsh, S. A., Banani, M. and Charkhkar, S. (2014): Phylogenetic analysis of $m g c 2$ gene of Mycoplasma gallisepticum isolates from broiler breeder flocks in Tehran province, Iran. Eur. J. Zool. Res. 3, 37-42.

Moretti, S. A., Boucher, C. E. and Bragg, R. R. (2013): Molecular characterisation of Mycoplasma gallisepticum genotypes from chickens in Zimbabwe and South Africa. South Afr. J. Sci. 109, $1-4$.

Rahimi, M. (2013): Food safety status of poultry meat and egg in Iran. World's Poult. Sci. J. 69, 401-406. doi:10.1017/S004393391300038X

Rasoulinezhad, S., Bozorgmehrifard, M. H., Hosseini, H., Sheikhi, N. and Charkhkar, S. (2017): Molecular detection and phylogenetic analysis of Mycoplasma gallisepticum from backyard and commercial turkey flocks in Iran. Vet. Res. Forum 8, 293.

Raviv, Z. and Ley, D. H. (2013): Mycoplasma gallisepticum infection. In: Swayne, D. E. (ed.) Diseases of Poultry. Wiley-Blackwell Press, Ames, IA. pp. 877-893.

Seifi, S. and Shirzad, M. R. (2012): Risk factors and seroprevalence of Mycoplasma gallisepticum infection in broiler breeder farms in Mazandaran province, north of Iran. Revue Med. Vet. 163, 215-218.

Tyler, K. D., Wang, G., Tyler, S. D. and Johnson, W. M. (1997): Factors affecting the reliability and reproducibility of amplification based DNA fingerprinting of representative bacterial pathogens. J. Clin. Microbiol. 35, 339-346.

Whithear, G. (1996): Control of avian mycoplasmosis by vaccination. Rev. Sci. Tech. 15, 1527-1553.

Younis, G. A., AbdElgawad, R. H., Elkenany, R. M. and Glal, A. F. (2018): Molecular identification and sequencing of Mycoplasma gallisepticum recovered from broilers in Egypt. Pakistan J. Biol. Sci. 21, 253-261. 